\title{
A NOTE ON COVER AND AVOIDANCE PROPERTIES IN SOLVABLE GROUPS
}

\author{
S. BAUMAN
}

In [1] Carter showed the existence within a finite solvable group of a conjugate class of nilpotent self normalizing subgroups. In [2] Gaschütz defined Carter's work in the more general setting of formation theory. In [1] Carter showed that his groups satisfy a cover and avoidance property with respect to suitable factors of $G$. This note will extend this result within the framework of formation theory.

All groups considered will be finite and solvable. All definitions appear in [1] or [2] and all notations will be standard save where explicit definitions are given. $\mathfrak{F}$ will be a formation whose $\mathfrak{F}$-groups may or may not exist. $G(\mathfrak{F})$ will be the unique normal subgroup of $G$ minimal with respect to the property that $G / G(\mathfrak{F}) \in \mathfrak{F}$.

Definition. If $M<G$, a series $\langle 1\rangle=G_{0} \triangleleft G_{1} \triangleleft \cdots \triangleleft G_{n}=G$ is called an $M$-series of $G$ if $G_{i}$ is normalized by $M$ and $G_{i} / G_{i-1}$ is a nontrivial irreducible $M$-factor.

THEOREM 1. If $F$ is an $\mathfrak{F}$-group of $G$ and $\left\{G_{i}\right\}, 0 \leqq i \leqq n$ is any $F$ series of $G$ then $F$ covers $G_{i} / G_{i-1}$ if and only if $F G_{i} / G_{i-1} \in \mathfrak{F}$.

Proof. Suppose $F G_{i} / G_{i-1} \in \mathcal{F}$ and $F$ avoids $G_{i} / G_{i-1}$. Since $\left(F \cap G_{i}\right) G_{i-1}=G_{i-1}$ we have $F \cap G_{i}<G_{i-1}$ and $F \cap G_{i}=F \cap G_{i-1}$. On the other hand since $F$ is an $\mathfrak{F}$-group, $F<F G_{i}$ and $F G_{i} / G_{i-1} \in \mathfrak{F}$ we get $F G_{i-1}=F G_{i}$. Since $G_{i-1}$ is proper in $G_{i}$ we get a contradiction to $F \cap G_{i}=F \cap G_{i-1}$. Suppose $F G_{i} / G_{i-1} \notin \mathfrak{F}$ and $F$ covers $G_{i} / G_{i-1}$. We have $F G_{i}=F G_{i-1}$. Thus $F G_{i} / G_{i-1}=F G_{i} / G_{i-1} \cong F / F \cap G_{i-1} \in \mathfrak{F}$. This is a contradiction.

What is more interesting is that this cover avoidance property actually characterizes the $\mathfrak{F}$-groups of $G$.

THEOREM 2. ${ }^{1}$ If $G$ is finite solvable and $\left\{G_{i}\right\}$ is an $M$-series of $G$ such that $M$ covers $G_{i} / G_{i-1}$ if and only if $M G_{i} / G_{i-1} \in \mathfrak{F}$ then $M$ is an ₹-group of $G$.

Proof. The proof will be by induction on $(G: M)|G|$. If $(G: M)|G|$ $=1$ then there is nothing to prove. Thus we may assume $(G: M)|G|$ $>1$. If $M=G$ then by hypothesis $M$ covers all factors and thus $M G_{1} / G_{0}=G \in \mathfrak{F}$. Thus $M$ is an $\mathfrak{F}$-group of $G$. If $M \neq G$ then $M$ does

Received by the editors September 25, 1967.

1 The author wishes to thank Dr. Dieter Blessenohl (Kiel) for supplying the proof of Theorem 2. His proof replaced a considerably longer proof of the author. 
not cover all the factors of the series $\left\{G_{i}\right\}$. Choose $t$ maximal so that $M$ does not cover $G_{t} / G_{t-1}$. Then it follows that $G=M G_{t}, G_{t} \unlhd G$, $G_{t-1} \unlhd G, G / G_{t} \in \mathfrak{F}$ and $G / G_{t-1} \notin \mathfrak{F}$. In particular $M G_{t-1}<G$. It is easy to see that $\left\{\Lambda_{i}\right\}$ where $\Lambda_{i}=G_{i} \cap M G_{t-1}$ forms an $M$-series of $M G_{t-1}$ satisfying the hypothesis of the theorem. Also the homomorphic images of $\left\{G_{i}\right\}$ show that $M G_{t-1} / G_{t-1}$ satisfies the hypotheses of the theorem in $G / G_{t-1}$. If $\left|G_{t-1}\right|>1$ then induction on $M$ in $M G_{t-1}$ and $M G_{t-1} / G_{t-1}$ in $G / G_{t-1}$ yields that $M$ is an $\mathfrak{F}$-group in $M G_{t-1}$ and $M G_{t-1} / G_{t-1}$ is an $\mathfrak{F - g r o u p ~ i n ~} G / G_{t-1}$. By [2, Lemma 2.3], it follows that $M$ is an $\mathfrak{F}$-group of $G$. Thus $\left|G_{t-1}\right|=1$ and $G_{t}$ is a minimal normal subgroup of $G$. It follows that $M$ is maximal in $G$ and $M \cap G_{1}=\langle 1\rangle$. Thus since $G / G_{1} \in \mathfrak{F}$ and $G \notin \mathfrak{F}$ we have that $G_{1}$ is precisely $G(\mathfrak{F})$. It follows that $M$ is an $\mathfrak{F}$-group of $G$.

To bring Carter's cover avoidance property under our theorem we prove

Theorem 3. Suppose $M<G$ and $M$ is nilpotent. Let $H / K$ be an irreducible $M$-factor of $G$. Then $M H / K$ is nilpotent if and only if $H / K$ is $M$-central.

Proof. If $M H / K$ is nilpotent since $H / K$ is minimal normal in $M H / K$ we have that $H / K<Z(M H / K)$. Thus $[M, H]<K$ or $H / K$ is $M$-central. If $H / K$ is $M$-central then $M H / K$ is the product of two normal nilpotent groups $M K / K$ and $H / K$. Thus $M H / K$ is nilpotent.

\section{REFERENCES}

1. R. Carter, Nilpotent self normalizing subgroups of solvable groups, Math. Z. 75 (1961), 136-139.

2. W. Gaschütz, Zur theorie der endlichen aufiösbaren Gruppen, Math. Z. 80 (1963), 300-305.

UNIVERSITY OF WISCONSIN 